\title{
Robust Synchronization of Incommensurate Fractional-Order Chaotic Systems via Second-Order Sliding Mode Technique
}

\author{
Hua Chen, ${ }^{1}$ Wen Chen, ${ }^{2,3}$ Binwu Zhang, ${ }^{1}$ and Haitao Cao ${ }^{1}$ \\ ${ }^{1}$ Mathematics and Physics Department, Hohai University, Changzhou Campus, Changzhou 213022, China \\ ${ }^{2}$ College of Mechanics and Materials, Hohai University, Nanjing 210098, China \\ ${ }^{3}$ State Key Laboratory of Hydrology-Water Resources and Hydraulic Engineering, Hohai University, Nanjing 210098, China
}

Correspondence should be addressed to Hua Chen; chenhua112@163.com

Received 21 February 2013; Revised 15 June 2013; Accepted 23 June 2013

Academic Editor: Kai Diethelm

Copyright (C) 2013 Hua Chen et al. This is an open access article distributed under the Creative Commons Attribution License, which permits unrestricted use, distribution, and reproduction in any medium, provided the original work is properly cited.

\begin{abstract}
A second-order sliding mode (SOSM) controller is proposed to synchronize a class of incommensurate fractional-order chaotic systems with model uncertainties and external disturbances. Based on the chattering free SOSM control scheme, it can be rigorously proved that the dynamics of the synchronization error is globally asymptotically stable by using the Lyapunov stability theorem. Finally, numerical examples are provided to illustrate the effectiveness of the proposed controller design approach.
\end{abstract}

\section{Introduction}

For the last few decades, the study of fractional-order control systems has attracted increasing interest (see, e.g., [1-7] and the references therein), where the system equations were described by the so-called fractional derivatives and integrals (for the introduction to this theory see $[1,8]$ ). Because fractional derivatives provide an excellent instrument for the description of memory and hereditary properties of various materials and processes, then the advantages of using the fractional order model are that we have more degrees of freedom in the model and that a "memory" is included in the model. The modeling of dynamical systems by using the means of the fractional calculus has been reported in many engineering areas such as signal processing [9], electromagnetism [10], mechanics [11-14], image processing [15], bioengineering [16], automatic control [17, 18], and robotics $[19,20]$. Among the existed literatures on the dynamics of fractional-order differential systems, it has been demonstrated that some fractional-order systems can behave chaotically or hyperchaotically [21-25].

Due to the existence of chaos in real practical systems and many potential applications in physics and engineering, the study of synchronizing/stabilizing chaotic/hyperchaotic systems has attracted considerable interests in the past decades [26-32]. Several methods have been proposed to achieve chaos synchronization. One of the methods is based on the sliding mode control (SMC) approach [27, 30, 31, 3336]. The main feature of the SMC is to switch the control law to drive the states of the system from the initial states onto some predefined sliding surface in a finite time. The system on the sliding surface has desired properties such as stability, disturbance rejection capability, and tracking ability [34].

In general, the traditional sliding mode control is of the first order. And there exists an inevitable drawback when applying such standard SMC, that is the so-called chattering phenomenon, namely, the occurrence of undesirable highfrequency vibrations of the system variables which are caused by the discontinuous high-frequency nature of first-order sliding mode control signals. In order to improve the control accuracy and reduce the undesired chattering effect by removing the controller discontinuity while keeping similar properties of robustness analogous as those featured by the conventional first-order sliding mode approach, the second(and higher) order sliding mode control method is proposed [37-40]. However, to the authors' knowledge, there are few researches on the fractional-order system using the SOSM control approach so far.

Motivated by the above discussions, this article considers the robust synchronization problem for a class of uncertain 
incommensurate fractional-order chaotic systems raised by Aghababa in [41]. A chattering free SOSM controller is presented in the presence of model uncertainties and external disturbance.

The structure of this paper is as follows: Section 2 recalls some preliminaries on fractional calculus and gives the statement of the problem considered in this paper. Section 3 provides the SOSM controller together with the respective Lyapunov-based stability analysis. Section 4 illustrates some simulation results. Finally, a conclusion is drawn in Section 5.

\section{Preliminaries and Problem Statement}

2.1. Basic Definitions of Fractional Calculus. There are many ways to define the fractional integral and derivative. Two definitions, Riemann-Liouville definition and Caputo definition, are generally used in recent literatures.

Definition 1 (see [1]). The $\alpha$ th-order Riemann-Liouville fractional integration of function $f(t)$ is given by

$$
t_{0} I_{t}^{\alpha} f(t)=\frac{1}{\Gamma(\alpha)} \int_{t_{0}}^{t} \frac{f(\tau)}{(t-\tau)^{1-\alpha}} d \tau,
$$

where $\Gamma(\alpha)$ is the Gamma function and $t_{0}$ is the initial time.

Definition 2 (see [1]). Letting $n-1<\alpha \leq n, n \in N$, the Riemann-Liouville fractional derivative of order $\alpha$ of function $f(t)$ is defined as follows:

$$
\begin{aligned}
t_{0} D_{t}^{\alpha} f(t) & =\frac{d^{\alpha} f(t)}{d t^{\alpha}} \\
& =\frac{1}{\Gamma(n-\alpha)} \frac{d^{n}}{d t^{n}} \int_{t_{0}}^{t} \frac{f(\tau)}{(t-\tau)^{\alpha-n+1}} d \tau \\
& =\frac{d^{n}}{d t^{n}} I^{n-\alpha} .
\end{aligned}
$$

Definition 3 (see [1]). The Caputo fractional derivative of order $\alpha$ of a continuous function $f(t)$ is defined as follows:

$$
{ }_{t_{0}} D_{t}^{\alpha} f(t)= \begin{cases}\frac{1}{\Gamma(n-\alpha)} \int_{t_{0}}^{t} \frac{f^{(n)}(\tau)}{(t-\tau)^{\alpha-n+1}} d \tau, & n-1<\alpha<n, \\ \frac{d^{n} f(t)}{d t^{n}}, & \alpha=n,\end{cases}
$$

where $n$ is the smallest integer number, larger than $\alpha$.

Lemma 4 (see [42]). Consider the system

$$
\dot{x}(t)=f(x(t)), \quad f(0)=0, \quad x(t) \in R^{n},
$$

where $f: D \rightarrow R^{n}$ is continuous on an open neighborhood $D \subset R^{n}$. Suppose there exists a continuous differential positivedefinite function $V(x(t)): D \rightarrow R$, real numbers $p>0,0<$ $\eta<1$, such that

$$
\dot{V}(x(t))+p V^{\eta}(x(t)) \leq 0, \quad \forall x(t) \in D .
$$

Then, the origin of system (4) is a locally finite-time stable equilibrium, and the settling time, depending on the initial state $x(0)=x_{0}$, satisfies $T\left(x_{0}\right) \leq V^{1-\eta}\left(x_{0}\right) / p(1-\eta)$. In addition, if $D=R^{n}$ and $V(x(t))$ is also radially unbounded, then the origin is a globally finite-time stable equilibrium of system (4).

Lemma 5 (see [43]). Consider a vector signal $z(t) \in R^{m}$. Let $\alpha \in(0,1)$. If there exists $t_{1}<\infty$ such that $I^{\alpha} z(t)=0, \forall t \geq t_{1}$, then $\lim _{t \rightarrow \infty} z(t)=0$.

2.2. Problem Statement. Consider the following $n$-dimensional uncertain incommensurate fractional-order chaotic/ hyperchaotic slave system:

$$
\begin{aligned}
& D^{q_{1}} x_{1}(t)=f_{1}(X, t)+\Delta f_{1}(X)+d_{1}^{f}(t)+u_{1}(t), \\
& D^{q_{2}} x_{2}(t)=f_{2}(X, t)+\Delta f_{2}(X)+d_{2}^{f}(t)+u_{2}(t),
\end{aligned}
$$

$$
D^{q_{n}} x_{n}(t)=f_{n}(X, t)+\Delta f_{n}(X)+d_{n}^{f}(t)+u_{n}(t),
$$

where $q_{i} \in(0,1), i=1,2, \ldots, n$, is the order of the system, $X(t)=\left[x_{1}(t), x_{2}(t), \ldots, x_{n}(t)\right]^{T} \in R^{n}$ is the state vector, $f_{i}(X, t) \in R, i=1,2, \ldots, n$, is a given nonlinear function of $X$ and $t, \Delta f_{i}(X) \in R, i=1,2, \ldots, n$, and $d_{i}^{f}(t) \in R, i=$ $1,2, \ldots, n$, denote unknown mode uncertain and external disturbances of the system, respectively, and $u_{i}(t) \in R$ is the control input.

Suppose the master system can be described as

$$
\begin{gathered}
D^{q_{1}} y_{1}(t)=g_{1}(Y, t)+\Delta g_{1}(Y)+d_{1}^{g}(t), \\
D^{q_{2}} y_{2}(t)=g_{2}(Y, t)+\Delta g_{2}(Y)+d_{2}^{g}(t), \\
\vdots \\
D^{q_{n}} y_{n}(t)=g_{n}(Y, t)+\Delta g_{n}(Y)+d_{n}^{g}(t),
\end{gathered}
$$

where $Y(t)=\left[y_{1}(t), y_{2}(t), \ldots, y_{n}(t)\right]^{T} \in R^{n}$ is the state vector, $g_{i}(Y, t) \in R, i=1,2, \ldots, n$, is a given nonlinear function of $Y$ and $t, \Delta g_{i}(Y) \in R, i=1,2, \ldots, n$, and $d_{i}^{g}(t) \in R, i=$ $1,2, \ldots, n$, denote unknown mode uncertain and external disturbances of the system, respectively.

We define the chaos synchronization problem as follows: design an appropriate controller $u_{i}(t), i=1,2,3, \ldots, n$, for the slave system (6) such that its state trajectories track the state trajectories of the master system (7) asymptotically.

Remark 6. If $q_{i}=q, i=1,2, \ldots, n$, then systems (6) and (7) are called commensurate fractional-order chaotic systems. The finite-time synchronization between (6) and (7) with the same fractional orders has been addressed in [43] by using a discontinuous terminal sliding mode control method; this paper considers an SOSM controller design for synchronizing the incommensurate fractional-order system.

Assumption 7. The uncertainty terms $\Delta f_{i}(X), \Delta g_{i}(Y)$, the external disturbances $d_{i}^{f}(t)$ and $d_{i}^{g}(t), i=1,2, \ldots, n$, are 
derivable, and the bounds of their derivatives are known positive constants $\gamma_{i}^{\Delta f_{d}}, \gamma_{i}^{\Delta g_{d}}, \delta_{i}^{f_{d}}$, and $\delta_{i}^{g_{d}}$ :

$$
\begin{aligned}
\left|\frac{d}{d t}\left(\Delta f_{i}(X)\right)\right| & \leq \gamma_{i}^{\Delta f_{d}}, \\
\left|\frac{d}{d t}\left(\Delta g_{i}(Y)\right)\right| & \leq \gamma_{i}^{\Delta g_{d}}, \\
\left|\frac{d}{d t}\left(d_{i}^{f}(t)\right)\right| & \leq \delta_{i}^{f_{d}}, \\
\left|\frac{d}{d t}\left(d_{i}^{g}(t)\right)\right| & \leq \delta_{i}^{g_{d}} .
\end{aligned}
$$

Remark 8. In order to design a chattering free second-order sliding mode controller, the smoothness hypotheses of the uncertainty and external disturbances terms are required as in Assumption 7, which is not necessary with the first-order sliding mode control approach. Indeed, this can be seen as a standard assumption when using second-order sliding mode technique [43].

Define the synchronization error as

$$
\begin{aligned}
E(t)= & Y(t)-X(t) \\
= & {\left[y_{1}(t), y_{2}(t), \ldots, y_{n}(t)\right]^{T} } \\
& -\left[x_{1}(t), x_{2}(t), \ldots, x_{n}(t)\right]^{T} \\
= & {\left[e_{1}(t), e_{2}(t), \ldots, e_{n}(t)\right]^{T} . }
\end{aligned}
$$

Consequently, the synchronization error dynamics is obtained as follows:

$$
\begin{aligned}
D^{q_{1}} e_{1}(t)= & g_{1}(Y, t)+\Delta g_{1}(Y)+d_{1}^{g}(t)-f_{1}(X, t) \\
& -\Delta f_{1}(X)-d_{1}^{f}(t)-u_{1}(t), \\
D^{q_{2}} e_{2}(t)= & g_{2}(Y, t)+\Delta g_{2}(Y)+d_{2}^{g}(t)-f_{2}(X, t) \\
& -\Delta f_{2}(X)-d_{2}^{f}(t)-u_{2}(t), \\
& \vdots \\
D^{q_{n}} e_{n}(t)= & g_{n}(Y, t)+\Delta g_{n}(Y)+d_{n}^{g}(t)-f_{n}(X, t) \\
& -\Delta f_{n}(X)-d_{n}^{f}(t)-u_{n}(t) .
\end{aligned}
$$

The control task is to design a chattering free secondorder sliding mode controller $u_{i}(t), i=1,2,3, \ldots, n$, such that the synchronization error system (10) can be stabilized to zero as time goes to infinity.

Remark 9. It is clear that if $Y(t)=0$, then the synchronization problem is transformed to the stabilization problem of the fractional-order uncertain chaotic system (6).

\section{Main Results}

To design a sliding mode controller, there are two steps. Firstly, a sliding surface should be constructed that represents a desired system dynamics. Secondly, a switching control law should be developed such that a sliding mode exists on every point of the sliding surface, and any states outside the surface are driven to reach the surface in a finite time [44].

In this paper, as a choice, we propose an integral type sliding surface as follows:

$$
\begin{gathered}
S(t)=\left[s_{1}(t), s_{2}(t), \ldots, s_{n}(t)\right]^{T}=0, \\
s_{i}(t)=I^{1-q_{i}} e_{i}(t), \quad i=1,2, \ldots, n .
\end{gathered}
$$

According to sliding mode control (SMC) method, when in the sliding mode, the switching surface and its derivative must satisfy the following conditions:

$$
S(t)=0, \quad \dot{S}(t)=0,
$$

from which, one can obtain the so-called equivalent control and then derive the sliding mode controller.

But, in this paper, different from the traditional sliding mode control, the SOSM controller to be designed will drive all the states of sliding variables $s_{i}(t)$ to zero in a finite time; then, by using Lemma 5 , one has

$$
\lim _{t \rightarrow \infty} e_{i}(t)=0, \quad \forall i \in\{1,2, \ldots, n\} .
$$

Next, we will give the main results.

Theorem 10. Under Assumption 7, consider the uncertain fractional-order chaotic synchronization error system (10) and the sliding surface (11) and take the following SOSM control law:

$$
\begin{aligned}
u_{i}(t)= & g_{i}(Y, t)-f_{i}(X, t)+k_{i_{1}} s_{i}(t) \\
& +k_{i_{2}}\left|s_{i}(t)\right|^{1 / 2} \operatorname{sgn}\left(s_{i}(t)\right)-w_{i}(t), \\
& \dot{w}_{i}(t)=-k_{i_{3}} \operatorname{sgn}\left(s_{i}(t)\right),
\end{aligned}
$$

where $i=1,2, \ldots, n$, and sgn is the sign function; $k_{i_{1}}, k_{i_{2}}, k_{i_{3}}>$ 0 denote the design parameters satisfying that

$$
\begin{gathered}
k_{i_{3}}>k_{i_{2}}^{2}, \\
\frac{1}{2} k_{i_{2}} \min \left\{5 k_{i_{1}}^{2}, k_{i_{3}}+\frac{1}{2} k_{i_{2}}^{2}+\frac{1}{2}-\sqrt{\left(k_{i_{3}}+\frac{1}{2} k_{i_{2}}^{2}+\frac{1}{2}\right)^{2}-2 k_{i_{3}}}\right\} \\
>\left(\gamma_{i}+\delta_{i}\right) \max \left\{k_{i_{2}}, k_{i_{1}}, 2\right\},
\end{gathered}
$$

where $\gamma_{i}=\gamma_{i}^{\Delta f_{d}}+\gamma_{i}^{\Delta g_{d}}, \delta_{i}=\delta_{i}^{f_{d}}+\delta_{i}^{g_{d}}$. Then the closed-loop system of (10) is globally and asymptotically stable.

Proof. According to Definition 2, we have

$$
\begin{aligned}
\dot{s}_{i}(t)= & \frac{d}{d t}\left(I^{1-q_{i}} e_{i}(t)\right)=D^{q_{i}} e_{i}(t) \\
= & g_{i}(Y, t)+\Delta g_{i}(Y)+d_{i}^{g}(t)-f_{i}(X, t) \\
& -\Delta f_{i}(X)-d_{i}^{f}(t)-u_{i}(t) .
\end{aligned}
$$


Substituting (14) into the previous equation, it yields

$$
\begin{aligned}
\dot{s}_{i}(t)= & -k_{i_{1}} s_{i}(t)-k_{i_{2}}\left|s_{i}(t)\right|^{1 / 2} \operatorname{sgn}\left(s_{i}(t)\right) \\
& +w_{i}(t)+d_{i}(t) \\
& \dot{w}_{i}(t)=-k_{i_{3}} \operatorname{sgn}\left(s_{i}(t)\right)
\end{aligned}
$$

where $d_{i}(t)=\Delta g_{i}(Y)+d_{i}^{g}(t)-\Delta f_{i}(X)-d_{i}^{f}(t)$. From Assumption 7, one has

$$
\begin{aligned}
\left|\frac{d}{d t}\left(d_{i}(t)\right)\right| \leq & \left|\frac{d}{d t}\left(\Delta g_{i}(Y)\right)\right|+\left|\frac{d}{d t}\left(d_{i}^{g}(t)\right)\right| \\
& +\left|\frac{d}{d t}\left(\Delta f_{i}(X)\right)\right|+\left|\frac{d}{d t}\left(d_{i}^{f}(t)\right)\right| \\
\leq & \gamma_{i}^{\Delta f_{d}}+\gamma_{i}^{\Delta g_{d}}+\delta_{i}^{f_{d}}+\delta_{i}^{g_{d}},
\end{aligned}
$$

which implies that

$$
\left|\frac{d}{d t}\left(d_{i}(t)\right)\right| \leq \gamma_{i}+\delta_{i} .
$$

Letting $z_{i}(t)=w_{i}(t)+d_{i}(t)$, then system (17) can be rewritten as

$$
\begin{gathered}
\dot{s}_{i}(t)=-k_{i_{1}} s_{i}(t)-k_{i_{2}}\left|s_{i}(t)\right|^{1 / 2} \operatorname{sgn}\left(s_{i}(t)\right)+z_{i}(t), \\
\dot{z}_{i}(t)=-k_{i_{3}} \operatorname{sgn}\left(s_{i}(t)\right)+\frac{d}{d t}\left(d_{i}(t)\right) .
\end{gathered}
$$

Selecting a Lyapunov function for system (20),

$$
\begin{array}{r}
V_{i}(t)=2 k_{i_{3}}\left|s_{i}(t)\right|+\frac{1}{2} z_{i}^{2}(t) \\
+\frac{1}{2}\left[k_{i_{2}}\left|s_{i}(t)\right|^{1 / 2} \operatorname{sgn}\left(s_{i}(t)\right)\right. \\
\left.+k_{i_{1}} s_{i}(t)-z_{i}(t)\right]^{2}
\end{array}
$$

which can also be written as a quadratic form $V_{i}(t)=$ $\zeta_{i}^{T}(t) P_{i} \zeta_{i}(t)$, where

$$
\begin{aligned}
\zeta_{i}(t)= & {\left[\left|s_{i}(t)\right|^{1 / 2} \operatorname{sgn}\left(s_{i}(t)\right) s_{i}(t) z_{i}(t)\right]^{T}, } \\
P_{i} & =\frac{1}{2}\left[\begin{array}{ccc}
\left(4 k_{i_{3}}+k_{i_{2}}^{2}\right) & k_{i_{1}} k_{i_{2}} & -k_{i_{2}} \\
k_{i_{1}} k_{i_{2}} & k_{i_{1}}^{2} & -k_{i_{1}} \\
-k_{i_{2}} & -k_{i_{1}} & 2
\end{array}\right] .
\end{aligned}
$$

It is obvious that $V_{i}(t)$ is continuous but is not differentiable at $s_{i}(t)=0$; it is positive and radially unbounded if $k_{i_{3}}>0$; that is,

$$
\lambda_{\min }\left\{P_{i}\right\}\left\|\zeta_{i}(t)\right\|_{2}^{2} \leq V_{i}(t) \leq \lambda_{\max }\left\{P_{i}\right\}\left\|\zeta_{i}(t)\right\|_{2}^{2},
$$

where $\left\|\zeta_{i}(t)\right\|_{2}^{2}=\left|s_{i}(t)\right|+s_{i}^{2}(t)+z_{i}^{2}(t)$ is the Euclidean norm of $\zeta_{i}(t)$ and $\lambda_{\min }\left(P_{i}\right)$ and $\lambda_{\text {max }}\left(P_{i}\right)$ are the minimal eigenvalue and the largest eigenvalue of matrix $P_{i}$, respectively. Taking the time derivative of $V_{i}(t)$ along system (20), we have

$$
\begin{aligned}
& \dot{V}_{i}(t)=2 k_{i_{3}} \operatorname{sgn}\left(s_{i}(t)\right) \dot{s}_{i}(t)+z_{i}(t) \dot{z}_{i}(t) \\
& +\left(k_{i_{2}}\left|s_{i}(t)\right|^{1 / 2} \operatorname{sgn}\left(s_{i}(t)\right)+k_{i_{1}} s_{i}(t)-z_{i}(t)\right) \\
& \cdot\left(\frac{k_{i_{2}}}{2}\left|s_{i}(t)\right|^{-1 / 2} \dot{s}_{i}(t)+k_{i_{1}} \dot{s}_{i}(t)-\dot{z}_{i}(t)\right) \\
& =2 k_{i_{3}}\left[-k_{i_{1}}\left|s_{i}(t)\right|-k_{i_{2}}\left|s_{i}(t)\right|^{1 / 2}+z_{i}(t) \operatorname{sgn}\left(s_{i}(t)\right)\right] \\
& +z_{i}(t)\left[-k_{i_{3}} \operatorname{sgn}\left(s_{i}(t)\right)+\frac{d}{d t}\left(d_{i}(t)\right)\right] \\
& +\left(k_{i_{2}}\left|s_{i}(t)\right|^{1 / 2} \operatorname{sgn}\left(s_{i}(t)\right)+k_{i_{1}} s_{i}(t)-z_{i}(t)\right) \\
& \cdot\left[\frac{k_{i_{2}}}{2\left|s_{i}(t)\right|^{1 / 2}}\right. \\
& \times\left(-k_{i_{1}} s_{i}(t)-k_{i_{2}}\left|s_{i}(t)\right|^{1 / 2} \operatorname{sgn}\left(s_{i}(t)\right)+z_{i}(t)\right) \\
& +k_{i_{1}}\left(-k_{i_{1}} s_{i}(t)-k_{i_{2}}\left|s_{i}(t)\right|^{1 / 2} \operatorname{sgn}\left(s_{i}(t)\right)+z_{i}(t)\right) \\
& \left.+k_{i_{3}} \operatorname{sgn}\left(s_{i}(t)\right)-\frac{d}{d t}\left(d_{i}(t)\right)\right] \\
& =-2 k_{i_{1}} k_{i_{3}}\left|s_{i}(t)\right|-2 k_{i_{2}} k_{i_{3}}\left|s_{i}(t)\right|^{1 / 2} \\
& +2 k_{i_{3}} z_{i}(t) \operatorname{sgn}\left(s_{i}(t)\right)-k_{i_{3}} z_{i}(t) \operatorname{sgn}\left(s_{i}(t)\right) \\
& +z_{i}(t) \frac{d}{d t}\left(d_{i}(t)\right) \\
& +\left(k_{i_{2}}\left|s_{i}(t)\right|^{1 / 2} \operatorname{sgn}\left(s_{i}(t)\right)+k_{i_{1}} s_{i}(t)-z_{i}(t)\right) \\
& \cdot\left[-\frac{1}{2} k_{i_{1}} k_{i_{2}}\left|s_{i}(t)\right|^{1 / 2} \operatorname{sgn}\left(s_{i}(t)\right)-\frac{1}{2} k_{i_{2}}^{2} \operatorname{sgn}\left(s_{i}(t)\right)\right. \\
& +\frac{k_{i_{2}}}{2\left|s_{i}(t)\right|^{1 / 2}} z_{i}(t)-k_{i_{2}}^{2} s_{i}(t) \\
& -k_{i_{1}} k_{i_{2}}\left|s_{i}(t)\right|^{1 / 2} \operatorname{sgn}\left(s_{i}(t)\right)+k_{i_{1}} z_{i}(t) \\
& \left.+k_{i_{3}} \operatorname{sgn}\left(s_{i}(t)\right)-\frac{d}{d t}\left(d_{i}(t)\right)\right] \text {. }
\end{aligned}
$$

By a simple derivation, we have

$$
\begin{aligned}
\dot{V}_{i}(t)= & -2 k_{i_{1}} k_{i_{3}}\left|s_{i}(t)\right|-2 k_{i_{2}} k_{i_{3}}\left|s_{i}(t)\right|^{1 / 2} \\
& +k_{i_{3}} z_{i}(t) \operatorname{sgn}\left(s_{i}(t)\right)+z_{i}(t) \frac{d}{d t}\left(d_{i}(t)\right) \\
+ & \left(k_{i_{2}}\left|s_{i}(t)\right|^{1 / 2} \operatorname{sgn}\left(s_{i}(t)\right)+k_{i_{1}} s_{i}(t)-z_{i}(t)\right) \\
& \cdot\left[-\frac{3}{2} k_{i_{1}} k_{i_{2}}\left|s_{i}(t)\right|^{1 / 2} \operatorname{sgn}\left(s_{i}(t)\right)\right. \\
& -\left(\frac{1}{2} k_{i_{2}}^{2}-k_{i_{3}}\right) \operatorname{sgn}\left(s_{i}(t)\right)+\frac{k_{i_{2}}}{2\left|s_{i}(t)\right|^{1 / 2}} z_{i}(t) \\
& \left.-k_{i_{1}}^{2} s_{i}(t)+k_{i_{1}} z_{i}(t)-\frac{d}{d t}\left(d_{i}(t)\right)\right]
\end{aligned}
$$




$$
\begin{aligned}
& =-2 k_{i_{1}} k_{i_{3}}\left|s_{i}(t)\right|-2 k_{i_{2}} k_{i_{3}}\left|s_{i}(t)\right|^{1 / 2} \\
& +k_{i_{3}} z_{i}(t) \operatorname{sgn}\left(s_{i}(t)\right)+z_{i}(t) \frac{d}{d t}\left(d_{i}(t)\right) \\
& -\frac{3}{2} k_{i_{1}} k_{i_{2}}^{2}\left|s_{i}(t)\right|-k_{i_{2}}\left(\frac{1}{2} k_{i_{2}}^{2}-k_{i_{3}}\right)\left|s_{i}(t)\right|^{1 / 2} \\
& +\frac{1}{2} k_{i_{2}}^{2} z_{i}(t) \operatorname{sgn}\left(s_{i}(t)\right)-k_{i_{1}}^{2} k_{i_{2}}\left|s_{i}(t)\right|^{3 / 2} \\
& +k_{i_{1}} k_{i_{2}}\left|s_{i}(t)\right|^{1 / 2} \operatorname{sgn}\left(s_{i}(t)\right) z_{i}(t) \\
& -k_{i_{2}}\left|s_{i}(t)\right|^{1 / 2} \operatorname{sgn}\left(s_{i}(t)\right) \frac{d}{d t}\left(d_{i}(t)\right) \\
& -\frac{3}{2} k_{i_{1}}^{2} k_{i_{2}}\left|s_{i}(t)\right|^{3 / 2}-k_{i_{1}}\left(\frac{1}{2} k_{i_{2}}^{2}-k_{i_{3}}\right)\left|s_{i}(t)\right| \\
& +\frac{1}{2} k_{i_{1}} k_{i_{2}}\left|s_{i}(t)\right|^{1 / 2} z_{i}(t) \operatorname{sgn}\left(s_{i}(t)\right) \\
& -k_{i_{1}}^{3} s_{i}^{2}(t)+k_{i_{1}}^{2} s_{i}(t) z_{i}(t)-k_{i_{1}} s_{i}(t) \frac{d}{d t}\left(d_{i}(t)\right) \\
& +\frac{3}{2} k_{i_{1}} k_{i_{2}}\left|s_{i}(t)\right|^{1 / 2} \operatorname{sgn}\left(s_{i}(t)\right) z_{i}(t) \\
& +\left(\frac{1}{2} k_{i_{2}}^{2}-k_{i_{3}}\right) \operatorname{sgn}\left(s_{i}(t)\right) z_{i}(t) \\
& -\frac{k_{i_{2}}}{2\left|s_{i}(t)\right|^{1 / 2}} z_{i}^{2}(t)+k_{i_{1}}^{2} s_{i}(t) z_{i}(t) \\
& -k_{i_{1}} z_{i}^{2}(t)+z_{i}(t) \frac{d}{d t}\left(d_{i}(t)\right) .
\end{aligned}
$$

The previous formula can be simplified as

$$
\begin{aligned}
\dot{V}_{i}(t)= & -\left(k_{i_{1}} k_{i_{3}}+2 k_{i_{1}} k_{i_{2}}^{2}\right)\left|s_{i}(t)\right| \\
& -\left(k_{i_{2}} k_{i_{3}}+\frac{1}{2} k_{i_{3}}^{2}\right)\left|s_{i}(t)\right|^{1 / 2}+k_{i_{2}}^{2} z_{i}(t) \operatorname{sgn}\left(s_{i}(t)\right) \\
& +\left(2 z_{i}-k_{i_{2}}\left|s_{i}(t)\right|^{1 / 2} \operatorname{sgn}\left(s_{i}(t)\right)-k_{i_{1}} s_{i}(t)\right) \\
& \times \frac{d}{d t}\left(d_{i}(t)\right)-\frac{5}{2} k_{i_{1}}^{2} k_{i_{2}}\left|s_{i}(t)\right|^{3 / 2} \\
& +3 k_{i_{1}} k_{i_{2}}\left|s_{i}(t)\right|^{1 / 2} z_{i}(t) \operatorname{sgn}\left(s_{i}(t)\right)-k_{i_{1}}^{3} s_{i}^{2}(t) \\
& +k_{i_{1}}^{2} s_{i}(t) z_{i}(t)-\frac{k_{i_{2}}}{2\left|s_{i}(t)\right|^{1 / 2}} z_{i}^{2}(t)-k_{i_{1}} z_{i}^{2}(t) \\
= & -\left(k_{i_{1}} k_{i_{3}}+2 k_{i_{1}} k_{i_{2}}^{2}\right)\left|s_{i}(t)\right| \\
& +\left(2 z_{i}(t)-k_{i_{2}}\left|s_{i}(t)\right|^{1 / 2} \operatorname{sgn}\left(s_{i}(t)\right)-k_{i_{1}} s_{i}(t)\right) \\
& \times \frac{d}{d t}\left(d_{i}(t)\right)
\end{aligned}
$$

$$
\begin{aligned}
+3 k_{i_{1}} k_{i_{2}}\left|s_{i}(t)\right|^{1 / 2} z_{i}(t) \operatorname{sgn}\left(s_{i}(t)\right) & \\
-k_{i_{1}}^{3} s_{i}^{2}(t)+ & k_{i_{1}}^{2} s_{i}(t) z_{i}(t)-k_{i_{1}} z_{i}^{2}(t) \\
-\frac{1}{\left|s_{i}(t)\right|^{1 / 2}} & {\left[\left(k_{i_{2}} k_{i_{3}}+\frac{1}{2} k_{i_{2}}^{3}\right)\left|s_{i}(t)\right|\right.} \\
& -k_{i_{2}}^{2}\left|s_{i}(t)\right|^{1 / 2} z_{i}(t) \operatorname{sgn}\left(s_{i}(t)\right) \\
& \left.+\frac{5}{2} k_{i_{1}}^{2} k_{i_{2}} s_{i}^{2}(t)+\frac{1}{2} k_{i_{2}} z_{i}^{2}(t)\right] .
\end{aligned}
$$

Therefore, we can rewrite $\dot{V}_{i}(t)$ as

$$
\begin{aligned}
\dot{V}_{i}(t)= & -\zeta_{i}^{T}(t) Q_{i_{1}} \zeta_{i}(t)-\frac{1}{\left|s_{i}(t)\right|^{1 / 2}} \zeta_{i}^{T}(t) Q_{i_{2}} \zeta_{i}(t) \\
& +q_{i}^{T} \frac{d}{d t}\left(d_{i}(t)\right) \zeta_{i}(t)
\end{aligned}
$$

where

$$
\begin{gathered}
Q_{i_{1}}=\left[\begin{array}{ccc}
k_{i_{1}} k_{i_{3}}+2 k_{i_{1}} k_{i_{2}}^{2} & 0 & -\frac{3}{2} k_{i_{1}} k_{i_{2}} \\
0 & k_{i_{1}}^{3} & -\frac{1}{2} k_{i_{1}}^{2} \\
-\frac{3}{2} k_{i_{1}} k_{i_{2}} & -\frac{1}{2} k_{i_{1}}^{2} & k_{i_{1}}
\end{array}\right], \\
Q_{i_{2}}=\left[\begin{array}{ccc}
k_{i_{2}} k_{i_{3}}+\frac{1}{2} k_{i_{2}}^{3} & 0 & -\frac{1}{2} k_{i_{2}}^{2} \\
0 & \frac{5}{2} k_{i_{1}}^{2} k_{i_{2}} & 0 \\
-\frac{1}{2} k_{i_{2}}^{2} & 0 & \frac{1}{2} k_{i_{2}}
\end{array}\right],
\end{gathered}
$$

$q_{i}^{T}=\left[\begin{array}{lll}-k_{i_{2}} & -k_{i_{1}} & 2\end{array}\right]$. Next, we will prove that the matrixes $Q_{i_{1}}$ and $Q_{i_{2}}$ are positive definite.

For all $k_{i_{1}}, k_{i_{2}}, k_{i_{3}}>0$, let

$$
\widetilde{Q}_{i_{1}}=\frac{Q_{i_{1}}}{k_{i_{1}}}=\left[\begin{array}{ccc}
k_{i_{3}}+2 k_{i_{2}}^{2} & 0 & -\frac{3}{2} k_{i_{2}} \\
0 & k_{i_{1}}^{2} & -\frac{1}{2} k_{i_{1}} \\
-\frac{3}{2} k_{i_{2}} & -\frac{1}{2} k_{i_{1}} & 1
\end{array}\right] .
$$

Then, by simple calculations and from the first inequality of (15), we have

$$
\begin{gathered}
k_{i_{3}}+2 k_{i_{2}}^{2}>0, \quad\left(k_{i_{3}}+2 k_{i_{2}}^{2}\right) k_{i_{1}}^{2}>0, \\
\operatorname{det}\left(\widetilde{Q}_{i_{1}}\right)=\frac{3}{4} k_{i_{1}}^{2}\left(k_{i_{3}}-k_{i_{2}}^{2}\right)>0,
\end{gathered}
$$

which implies that $Q_{i_{1}}>0$. 
As for $Q_{i_{2}}$, by direct calculation, three positive eigenvalues of it can be obtained:

$$
\begin{gathered}
\lambda_{1}=\frac{5}{2} k_{i_{1}}^{2} k_{i_{2}} \\
\lambda_{2,3}=\left(k_{i_{2}} k_{i_{3}}+\frac{1}{2} k_{i_{2}}^{3}+\frac{1}{2} k_{i_{2}}\right. \\
\left. \pm \sqrt{\left(k_{i_{2}} k_{i_{3}}+\frac{1}{2} k_{i_{2}}^{3}+\frac{1}{2} k_{i_{2}}\right)^{2}-2 k_{i_{2}}^{2} k_{i_{3}}}\right) \times(2)^{-1}
\end{gathered}
$$

which means $Q_{i_{2}}>0$.

Noting that $\left|s_{i}(t)\right|^{1 / 2} \leq\left\|\zeta_{i}(t)\right\|_{2}$, according to (19) and (27), one has

$$
\begin{aligned}
\dot{V}_{i}(t) \leq & -\zeta_{i}^{T}(t) Q_{i_{1}} \zeta_{i}(t)-\frac{1}{\left|s_{i}(t)\right|^{1 / 2}} \zeta_{i}^{T}(t) Q_{i_{2}} \zeta_{i}(t) \\
& +\frac{1}{\left|s_{i}(t)\right|^{1 / 2}}\left(\gamma_{i}+\delta_{i}\right) q_{i}^{T}\left\|\zeta_{i}(t)\right\|_{2}^{2} \\
\leq & -\zeta_{i}^{T}(t) Q_{i_{1}} \zeta_{i}(t)-\frac{1}{\left|s_{i}(t)\right|^{1 / 2}} \zeta_{i}^{T}(t) Q_{i_{2}} \zeta_{i}(t) \\
& +\frac{\left(\gamma_{i}+\delta_{i}\right)}{\left|s_{i}(t)\right|^{1 / 2}} \max \left\{k_{i_{2}}, k_{i_{1}}, 2\right\}\left\|\zeta_{i}(t)\right\|_{2}^{2} .
\end{aligned}
$$

By using the second inequality of (15) and formula (31) results

$$
\lambda_{\min }\left\{Q_{i_{2}}\right\}=\lambda_{3}>\left(\gamma_{i}+\delta_{i}\right) \max \left\{k_{i_{2}}, k_{i_{1}}, 2\right\} .
$$

Hence, from (32), one has

$$
\begin{aligned}
\dot{V}_{i}(t) \leq & -\lambda_{\min }\left\{Q_{i_{1}}\right\}\left\|\zeta_{i}(t)\right\|_{2}^{2} \\
& -\frac{1}{\left|s_{i}(t)\right|^{1 / 2}}\left(\lambda_{\min }\left\{Q_{i_{2}}\right\}-\left(\gamma_{i}+\delta_{i}\right) \max \left\{k_{i_{2}}, k_{i_{1}}, 2\right\}\right) \\
& \times\left\|\zeta_{i}(t)\right\|_{2}^{2} .
\end{aligned}
$$

Because

$$
\begin{gathered}
\left\|\zeta_{i}(t)\right\|_{2}^{2}=\left|s_{i}(t)\right|+s_{i}^{2}(t)+z_{i}^{2}(t), \\
\lambda_{\min }\left\{P_{i}\right\}\left\|\zeta_{i}(t)\right\|_{2}^{2} \leq V_{i}(t) \leq \lambda_{\max }\left\{P_{i}\right\}\left\|\zeta_{i}(t)\right\|_{2}^{2},
\end{gathered}
$$

we have

$$
\begin{gathered}
\left|s_{i}(t)\right|^{1 / 2} \leq\left\|\zeta_{i}(t)\right\|_{2} \leq \frac{\sqrt{V_{i}(t)}}{\sqrt{\lambda_{\min }\left\{P_{i}\right\}}}, \\
\frac{V_{i}(t)}{\lambda_{\max }\left\{P_{i}\right\}} \leq\left\|\zeta_{i}(t)\right\|_{2}^{2} \leq \frac{V_{i}(t)}{\lambda_{\min }\left\{P_{i}\right\}} .
\end{gathered}
$$

Therefore, from (34), we have

$$
\begin{aligned}
\dot{V}_{i}(t) \leq & -\frac{\lambda_{\min }\left\{Q_{i_{1}}\right\}}{\lambda_{\max }\left\{P_{i}\right\}} V_{i}(t) \\
& -\left(\lambda_{\min }\left\{Q_{i_{2}}\right\}-\left(\gamma_{i}+\delta_{i}\right) \max \left\{k_{i_{2}}, k_{i_{1}}, 2\right\}\right) \\
& \times \frac{V_{i}(t)}{\lambda_{\max }\left\{P_{i}\right\}} \cdot \frac{\sqrt{\lambda_{\min }\left\{P_{i}\right\}}}{\sqrt{V_{i}(t)}} \\
\leq & -\frac{\sqrt{\lambda_{\min }\left\{P_{i}\right\}}}{\lambda_{\max }\left\{P_{i}\right\}} \\
& \times\left(\lambda_{\min }\left\{Q_{i_{2}}\right\}-\left(\gamma_{i}+\delta_{i}\right) \max \left\{k_{i_{2}}, k_{i_{1}}, 2\right\}\right) \sqrt{V_{i}(t)} .
\end{aligned}
$$

By Lemma 4 it follows easily that $V_{i}(t)$ and therefore $s_{i}(t)$, globally converge to zero in a finite time. According to the sliding surface dynamics (11) and Lemma 5, we obtain $e_{i}(t) \rightarrow 0$ as $t \rightarrow \infty$.

This completes the proof of Theorem 10 .

Remark 11. It is difficult to obtain all the possible solutions of nonlinear inequalities (15). However, in the process of selecting parameters, we observe that

$$
\begin{aligned}
k_{i_{3}}+\frac{1}{2} k_{i_{2}}^{2}+\frac{1}{2}+\sqrt{\left(k_{i_{3}}+\frac{1}{2} k_{i_{2}}^{2}+\frac{1}{2}\right)^{2}-2 k_{i_{3}}} & \frac{2 k_{i_{3}}}{k_{i_{3}}+(1 / 2) k_{i_{2}}^{2}+1 / 2+\sqrt{\left(k_{i_{3}}+(1 / 2) k_{i_{2}}^{2}+1 / 2\right)^{2}-2 k_{i_{3}}}} \\
\geq & \frac{2 k_{i_{3}}}{k_{i_{3}}+(1 / 2) k_{i_{3}}+1 / 2+\sqrt{\left(k_{i_{3}}+(1 / 2) k_{i_{3}}+1 / 2\right)^{2}}} \\
= & \frac{2 k_{i_{3}}}{3 k_{i_{3}}+1} .
\end{aligned}
$$

Therefore, if $\gamma_{i}+\delta_{i}<4$, we may present a set of feasible solutions of design parameters $k_{i_{1}}, k_{i_{2}}$, and $k_{i_{3}}$ step by step. First, choosing $k_{i_{1}}, k_{i_{2}}$ satisfies $\sqrt{2 / 15}<k_{i_{1}} \leq 2$ and $\sqrt[3]{2\left(\gamma_{i}+\delta_{i}\right)}<k_{i_{2}} \leq 2$. Next, select $k_{i_{3}}$ such that $k_{i_{2}}^{2}<k_{i_{3}} \leq$ $\left(\left(k_{i_{2}}^{3} / 2\left(\gamma_{i}+\delta_{i}\right)\right)-1\right) / 3$. Thus it is an easy task to get a group of appropriate design parameters in this way.

\section{Simulations}

A useful approximate numerical technique for solving the fractional differential equations has been developed by many researchers; see, for example, Diethelm et al. [45], which is the generalization of the Adams-Bashforth-Moulton algorithm. 

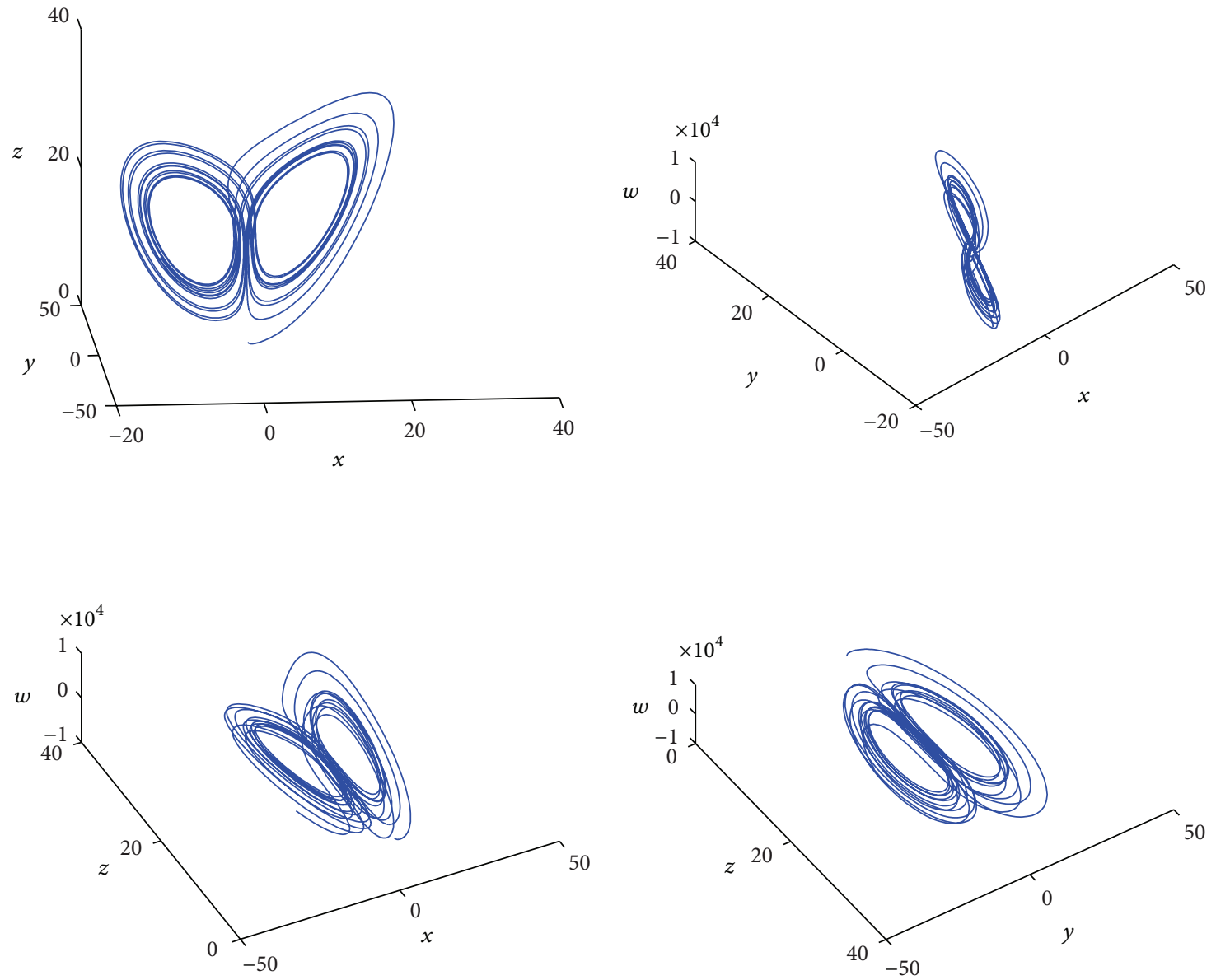

FIGURE 1: Response of the fractional-order hyperchaotic Chen system with respect to time.

Based on the numerical algorithms of fractional-order systems, we consider the simulation example for the synchronization problem of the uncertain fractional-order hyperchaotic Lorenz system as the slave system and the fractionalorder hyperchaotic Chen system as the master system [41].

Firstly, we describe the hyperchaos phenomenon in the fractional-order hyperchaotic Chen system and the fractional-order hyperchaotic Lorenz system, respectively. For simplicity, we consider the following systems:

$$
\begin{aligned}
& D^{q_{1}} y_{1}(t)=35\left(y_{2}(t)-y_{1}(t)\right)+y_{4}(t), \\
& D^{q_{2}} y_{2}(t)=7 y_{1}(t)+12 y_{2}(t)-y_{1}(t) y_{3}(t), \\
& D^{q_{3}} y_{3}(t)=y_{1}(t) y_{2}(t)-8 y_{3}(t), \\
& D^{q_{4}} y_{4}(t)=y_{2}(t) y_{3}(t)+0.3 y_{4}(t), \\
& D^{q_{1}} x_{1}(t)=10\left(x_{2}(t)-x_{1}(t)\right)+x_{4}(t), \\
& D^{q_{2}} x_{2}(t)=28 x_{1}(t)-x_{2}(t)-x_{1}(t) x_{3}(t), \\
& D^{q_{3}} x_{3}(t)=x_{1}(t) x_{2}(t)-\frac{8}{3} x_{3}(t), \\
& D^{q_{4}} x_{4}(t)=-x_{2}(t) x_{3}(t)-x_{4}(t),
\end{aligned}
$$

where we take the fractional orders $q_{1}=0.98, q_{2}=$ $0.96, q_{3}=0.97$, and $q_{4}=0.99$. Assume the initial conditions are $(0.2,0.3,1.5,-0.5)$ and $(0.1,0.2,-0.3,1.5)$. By using the numerical algorithm similar to [46], the time step is $0.005 \mathrm{~s}$. Figures 1 and 2 show the hyperchaotic phenomenon.

Next, we consider the synchronization simulations of these two uncertain fractional-order hyperchaotic systems; the first is hyperchaotic Lorenz system:

$$
\begin{aligned}
D^{q_{1}} x_{1}(t)= & 10\left(x_{2}(t)-x_{1}(t)\right)+x_{4}(t)+\Delta f_{1}(X) \\
& +d_{1}^{f}(t)+u_{1}(t), \\
D^{q_{2}} x_{2}(t)= & 28 x_{1}(t)-x_{2}(t)-x_{1}(t) x_{3}(t)+\Delta f_{2}(X) \\
& +d_{2}^{f}(t)+u_{2}(t), \\
D^{q_{3}} x_{3}(t)= & x_{1}(t) x_{2}(t)-\frac{8}{3} x_{3}(t)+\Delta f_{3}(X) \\
& +d_{3}^{f}(t)+u_{3}(t), \\
D^{q_{4}} x_{4}(t)= & -x_{2}(t) x_{3}(t)-x_{4}(t)+\Delta f_{4}(X) \\
& +d_{4}^{f}(t)+u_{4}(t) .
\end{aligned}
$$



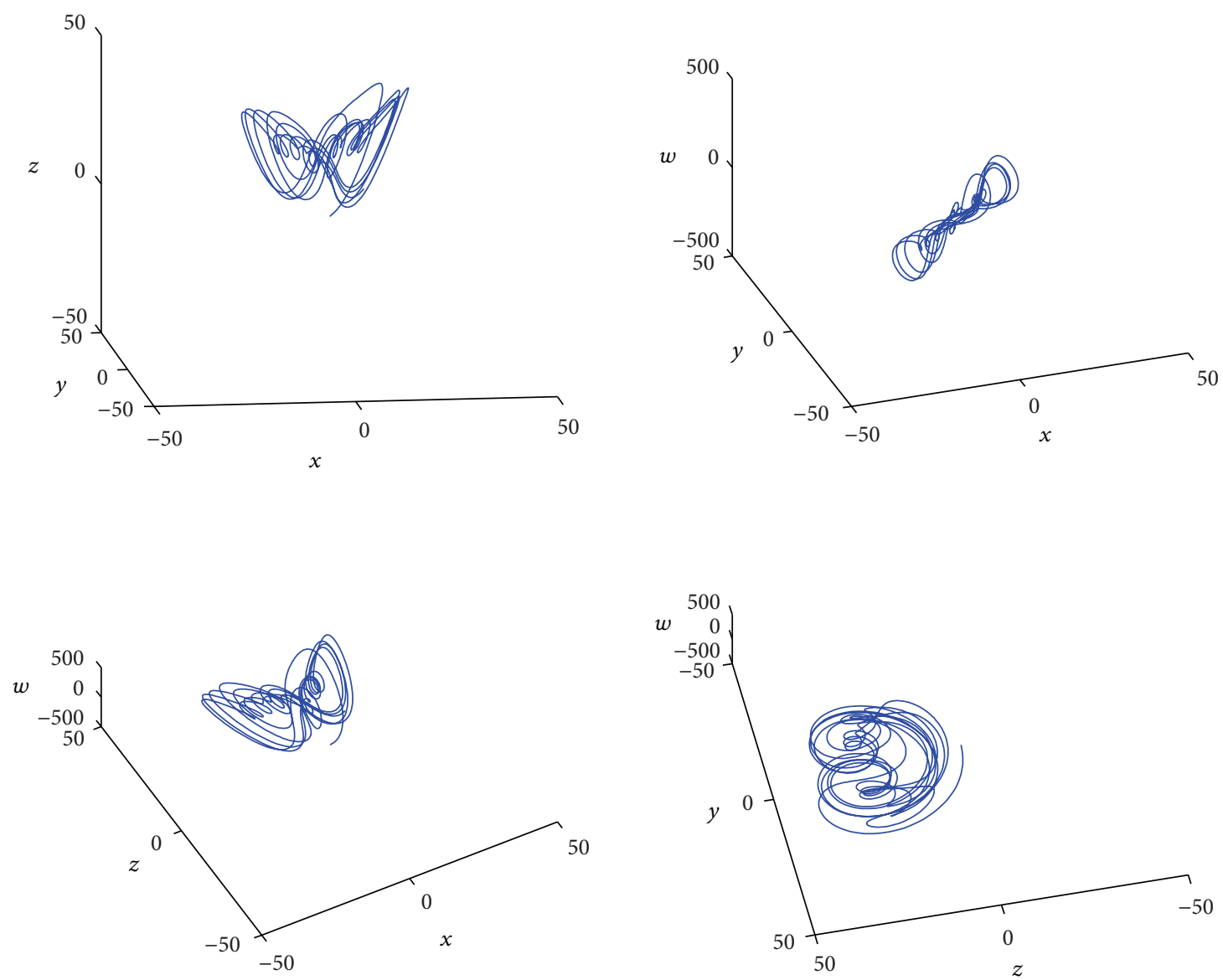

FIGURE 2: Response of the fractional-order hyperchaotic Lorenz system with respect to time.

The second is hyperchaotic Chen system:

$$
\begin{aligned}
D^{q_{1}} y_{1}(t)= & 35\left(y_{2}(t)-y_{1}(t)\right)+y_{4}(t)+\Delta g_{1}(Y)+d_{1}^{g}(t), \\
D^{q_{2}} y_{2}(t)= & 7 y_{1}(t)+12 y_{2}(t)-y_{1}(t) y_{3}(t)+\Delta g_{2}(Y) \\
& +d_{2}^{g}(t), \\
D^{q_{3}} y_{3}(t)= & y_{1}(t) y_{2}(t)-8 y_{3}(t)+\Delta g_{3}(Y)+d_{3}^{g}(t), \\
D^{q_{4}} y_{4}(t)= & y_{2}(t) y_{3}(t)+0.3 y_{4}(t)+\Delta g_{4}(Y)+d_{4}^{g}(t) .
\end{aligned}
$$

The uncertainty terms of systems (40) and (41) are selected as follows:

$$
\begin{gathered}
\Delta f_{1}+d_{1}^{f}=0.25 \cos 6 t-0.1 \sin t, \\
\Delta f_{2}+d_{2}^{f}=-0.2 \cos 2 t+0.15 \sin 3 t, \\
\Delta f_{3}+d_{3}^{f}=0.15 \sin 3 t-0.2 \cos t, \\
\Delta f_{4}+d_{4}^{f}=-0.3 \cos t-0.15 \cos t, \\
\Delta g_{1}+d_{1}^{g}=-0.15 \cos 4 t+0.2 \cos t, \\
\Delta g_{2}+d_{2}^{g}=0.1 \sin 4 t+0.2 \cos 2 t, \\
\Delta g_{3}+d_{3}^{g}=0.25 \sin 3 t-0.3 \cos 4 t, \\
\Delta g_{4}+d_{4}^{g}=0.15 \sin 5 t-0.1 \cos 2 t .
\end{gathered}
$$

As pointed out in $[47,48]$, to ensure the existence of chaos for the hyperchaotic Lorenz and Chen systems, the initial conditions of the slave and master systems are chosen as $x_{1}(0)=2, x_{2}(0)=-1, x_{3}(0)=3, x_{4}(0)=2, y_{1}(0)=3$, $y_{2}(0)=5, y_{3}(0)=-3$, and $y_{4}(0)=1$, respectively. By Remark 11, choose parameters $k_{i_{1}}=1.5, k_{i_{2}}=1.6$, and $k_{i_{3}}=2.5$.

According to (40) and (41), the synchronization error dynamics is described as

$$
\begin{aligned}
D^{q_{1}} e_{1}= & 35\left(y_{2}-y_{1}\right)+y_{4}-0.15 \cos 4 t+0.2 \cos t \\
& -10\left(x_{2}-x_{1}\right)-x_{4}-0.25 \cos 6 t+0.1 \sin t-u_{1}, \\
D^{q_{2}} e_{2}= & 7 y_{1}+12 y_{2}-y_{1} y_{3}+0.1 \sin 4 t+0.2 \cos 2 t \\
& -28 x_{1}+x_{2}+x_{1} x_{3}+0.2 \cos 2 t-0.15 \sin 3 t-u_{2}, \\
D^{q_{3}} e_{3}= & y_{1} y_{2}-8 y_{3}+0.25 \sin 3 t-0.3 \cos 4 t-x_{1} x_{2} \\
& +\frac{8}{3} x_{3}-0.15 \sin 3 t+0.2 \cos t-u_{3}, \\
D^{q_{4}} e_{4}= & y_{2} y_{3}+0.3 y_{4}+0.15 \sin 5 t-0.1 \cos 2 t+x_{2} x_{3} \\
& +x_{4}+0.3 \cos t+0.15 \cos t-u_{4},
\end{aligned}
$$

with the initial conditions being $e_{1}(0)=1, e_{2}(0)=6, e_{3}(0)=$ -6 , and $e_{4}(0)=-1$. Substituting the second-order sliding 

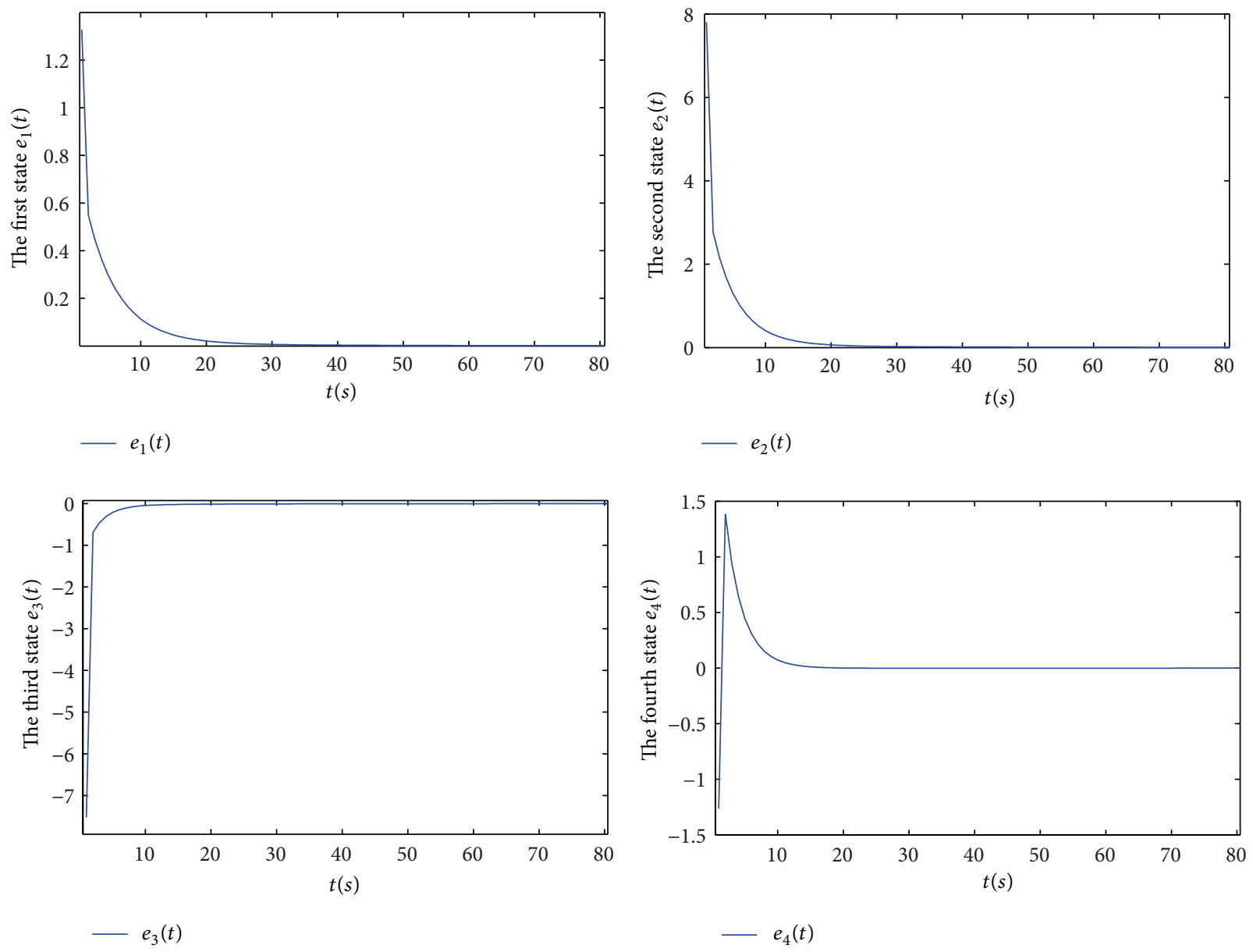

Figure 3: Response of the synchronization error $\left(e_{1}, e_{2}, e_{3}, e_{4}\right)$ with respect to time.

mode controller (14) into (43), we can obtain the closed-loop error system.

By using the numerical algorithm [45], with the sampling interval being $h=0.002 \mathrm{~s}$, next we present the simulation result to show the convergence of $e_{i}(t), i=1,2,3,4$.

From Figure 3, we observe that all the states of synchronization error system (43) converge to zero driven by the second-order sliding mode controller, which implies that the control approach is valid to address the robust synchronization problem for the uncertain hyperchaotic systems.

Remark 12. As given by Aghababa in [41], the uncertainty terms of systems (40) and (41) are chosen as bounded periodic function containing sine and cosine forms. Of course, other uncertain cases satisfying (8) can also be selected as the simulate examples.

Remark 13. As in [41], in this section, the fractional-order hyperchaotic Lorenz system and the fractional-order hyperchaotic Chen system are selected as slave system and the master system, respectively. In fact, there are many other fractional-order chaotic systems that can be considered; here we cannot discuss each case for lack of space.
Remark 14. In this section, we adopt the traditional numerical algorithm [45] for fractional-order system, as for the other method [46] with MATLAB implementation that can also be applied in our simulation section.

Remark 15. For the chaotic fractional systems, the orders should be lower than 3 , as for the hyperchaotic systems in our paper, even though the systems are of order $>3$, but, as pointed out in $[47,48]$, the existence of chaos can be guaranteed just as shown in Figures 1 and 2.

\section{Conclusion}

A second-order sliding mode controller is proposed in this article in order to address the synchronization problem for a class of uncertain fractional-order chaotic systems. The stability analysis is given based on the Lyapunov theorem; a simple numerical example is adopted to show the effectiveness of our control approach.

\section{Acknowledgments}

This paper was supported by the National Basic Research Program of China (973 Project, 2010CB832702), the National 
Science Funds for Distinguished Young Scholars (11125208), the 111 Project (B12032), the R\&D Special Fund for Public Welfare Industry (Hydrodynamics Project, 201101014), the Natural Science Foundation of China (11202066), the China Postdoctoral Science Foundation (2013M531263), and the Fundamental Research Funds for the Central Universities (2013B10114).

\section{References}

[1] I. Podlubny, Fractional Differential Equations, Academic Press, New York, NY, USA, 1999.

[2] R. Hilfer, Application of Fractional Calculus in Physics, World Science Publishing, Singapore, 2000.

[3] M. D. Ortigueira and J. A. Tenreiro Machado, "Fractional signal processing and applications," Signal Processing, vol. 83, no. 11, pp. 2285-2286, 2003.

[4] P. Lanusse, H. Benlaoukli, D. Nelson-Gruel, and A. Oustaloup, "Fractional-order control and interval analysis of SISO systems with time-delayed state," IET Control Theory \& Applications, vol. 2, no. 1, pp. 16-23, 2008.

[5] http://mechatronics.ece.usu.edu/foc/cdc02tw/cdrom/Lectures/ AppendixB/appendixB.pdf.

[6] I. Petráš, "Tuning and implementation methods for fractionalorder controllers," Fractional Calculus and Applied Analysis, vol. 15, no. 2, pp. 282-303, 2012.

[7] L. Dorcak, "Numerical models for the simulation of the fractional-order control systems," 2002, http://arxiv.org/abs/ math/0204108vl.

[8] K. B. Oldham and J. Spanier, The Fractional Calculus, Academic Press, New York, NY, USA, 1974.

[9] B. B. Mandelbrot and J. W. Van Ness, "Fractional Brownian motions, fractional noises and applications," SIAM Review, vol. 10, pp. 422-437, 1968.

[10] N. Engheta, "On fractional calculus and fractional multipoles in electromagnetism," IEEE Transactions on Antennas and Propagation, vol. 44, no. 4, pp. 554-566, 1996.

[11] D. Baleanu, A. K. Golmankhaneh, R. Nigmatullin, and A. K. Golmankhaneh, "Fractional Newtonian mechanics," Central European Journal of Physics, vol. 8, no. 1, pp. 120-125, 2010.

[12] H. G. Sun, W. Chen, H. Wei, and Y. Q. Chen, "A comparative study of constant-order and variable-order fractional models in characterizing memory property of systems," European Physical Journal, vol. 193, no. 1, pp. 185-192, 2011.

[13] H. Sun, Y. Chen, and W. Chen, "Random-order fractional differential equation models," Signal Processing, vol. 91, no. 3, pp. 525-530, 2011.

[14] W. Chen, J. Lin, and F. Wang, "Regularized meshless method for nonhomogeneous problems," Engineering Analysis with Boundary Elements, vol. 35, no. 2, pp. 253-257, 2011.

[15] A. Oustaloup, La Derivation Non Entiere: Theorie. Synthese et Application S, Editions Hermes, Paris, France, 1995.

[16] R. L. Magin, "Fractional calculus in bioengineering, part 3," Critical Reviews in Biomedical Engineering, vol. 32, no. 3-4, pp. 195-377, 2004.

[17] I. Podlubny, "Fractional-order systems and $P I^{\lambda} D^{\mu}$-controllers," IEEE Transactions on Automatic Control, vol. 44, no. 1, pp. 208214, 1999.

[18] M. S. Tavazoei, M. Haeri, S. Jafari, S. Bolouki, and M. Siami, "Some applications of fractional calculus in suppression of chaotic oscillations," IEEE Transactions on Industrial Electronics, vol. 55, no. 11, pp. 4094-4101, 2008.

[19] H. Linares, C. Baillot, A. Oustaloup, and C. Ceyral, "Generation of a fractional ground: application in robotics," in Proceedings of the International Congress IEE-Smc (CESA '96), Lille, France, July 1996.

[20] F. B. M. Duarte and J. A. Tenreiro Machado, "Chaotic phenomena and fractional-order dynamics in the trajectory control of redundant manipulators," Nonlinear Dynamics, vol. 29, no. 1-4, pp. 315-342, 2002.

[21] C. Li and G. Chen, "Chaos in the fractional order Chen system and its control," Chaos, Solitons \& Fractals, vol. 22, no. 3, pp. 549-554, 2004.

[22] L.-J. Sheu, H.-K. Chen, J.-H. Chen et al., "Chaos in the Newton-Leipnik system with fractional order," Chaos, Solitons \& Fractals, vol. 36, no. 1, pp. 98-103, 2008.

[23] W. M. Ahmad and J. C. Sprott, "Chaos in fractional-order autonomous nonlinear systems," Chaos, Solitons \& Fractals, vol. 16, no. 2, pp. 339-351, 2003.

[24] I. Grigorenko and E. Grigorenko, "Chaotic dynamics of the fractional Lorenz system," Physical Review Letters, vol. 91, no. 3, Article ID 034101, 4 pages, 2003.

[25] M.-F. Danca, "Chaotic behavior of a class of discontinuous dynamical systems of fractional-order," Nonlinear Dynamics, vol. 60, no. 4, pp. 525-534, 2010.

[26] M. M. Asheghan, M. T. H. Beheshti, and M. S. Tavazoei, "Robust synchronization of perturbed Chen's fractional-order chaotic system," Communications in Nonlinear Science and Numerical Simulation, vol. 16, pp. 1044-1051, 2011.

[27] M. P. Aghababa, S. Khanmohammadi, and G. Alizadeh, "Finitetime synchronization of two different chaotic systems with unknown parameters via sliding mode technique," Applied Mathematical Modelling, vol. 35, no. 6, pp. 3080-3091, 2011.

[28] R. Zhang and S. Yang, "Stabilization of fractional-order chaotic system via a single state adaptive-feedback controller," Nonlinear Dynamics, vol. 68, no. 1-2, pp. 45-51, 2012.

[29] S. Wang and Y. G. Yu, "Generalized projective synchronization of fractional order chaotic systems with different dimensions," Chinese Physics Letters, vol. 29, no. 2, Article ID 020505, 3 pages, 2012.

[30] C. Yin, S. Dadras, S. M. Zhang, and Y. Q. Chen, "Control of a novel class of fractional-order chaotic systems via adaptive sliding mode control approach," Applied Mathematical Modelling, vol. 37, no. 4, pp. 1607-2600, 2013.

[31] S. H. Hosseinnia, R. Ghaderi, A. Ranjbar N., M. Mahmoudian, and S. Momani, "Sliding mode synchronization of an uncertain fractional order chaotic system," Computers \& Mathematics with Applications, vol. 59, no. 5, pp. 1637-1643, 2010.

[32] X. J. Wu, H. T. Lu, and S. L. Shen, "Synchronization of a new fractional-order hyperchaotic system," Physics Letters A, vol. 373, no. 27-28, pp. 2329-2337, 2009.

[33] D. M. Senejohnny and H. Delavari, "Active sliding observer scheme based fractional chaos synchronization," Communications in Nonlinear Science and Numerical Simulation, vol. 17, no. 11, pp. 4373-4383, 2012.

[34] C. Yin, S. Dadras, and S.-M. Zhong, "Design an adaptive sliding mode controller for drive-response synchronization of two different uncertain fractional-order chaotic systems with fully unknown parameters," Journal of the Franklin Institute, vol. 349, no. 10, pp. 3078-3101, 2012. 
[35] D.-Y. Chen, Y.-X. Liu, X.-Y. Ma, and R.-F. Zhang, "Control of a class of fractional-order chaotic systems via sliding mode," Nonlinear Dynamics, vol. 67, no. 1, pp. 893-901, 2012.

[36] X. J. Wu, D. R. Lai, and H. T. Lu, "Generalized synchronization of the fractional-order chaos in weighted complex dynamical networks with nonidentical nodes," Nonlinear Dynamics, vol. 69, no. 1-2, pp. 667-683, 2012.

[37] G. Bartolini, L. Fridman, A. Pisano, and E. Usai, Eds., Modern Sliding Mode Control Theory. New Perspectives and Applications, vol. 375 of Lecture Note in Control and Information Sciences, Springer, Berlin, Germany, 2008.

[38] A. Levant, "Quasi-continuous high-order sliding-mode controllers," IEEE Transactions on Automatic Control, vol. 50, no. 11, pp. 1812-1816, 2005.

[39] A. Levant, "Principles of 2-sliding mode design," Automatica, vol. 43, no. 4, pp. 576-586, 2007.

[40] J. A. Moreno and M. Osorio, "A Lyapunov approach to secondorder sliding mode controllers and observers," in Proceedings of the 47th IEEE Conference on Decision and Control (CDC '08), pp. 2856-2861, December 2008.

[41] M. P. Aghababa, "Finite-time chaos control and synchronization of fractional-order nonautonomous chaotic (hyperchaotic) systems using fractional nonsingular terminal sliding mode technique," Nonlinear Dynamics, vol. 69, no. 1-2, pp. 247-261, 2012.

[42] S. P. Bhat and D. S. Bernstein, "Finite-time stability of continuous autonomous systems," SIAM Journal on Control and Optimization, vol. 38, no. 3, pp. 751-766, 2000.

[43] A. Pisano, M. R. Rapaić, Z. D. Jeličić, and E. Usai, "Sliding mode control approaches to the robust regulation of linear multivariable fractional-order dynamics," International Journal of Robust and Nonlinear Control, vol. 20, no. 18, pp. 2045-2056, 2010.

[44] S. Dadras and H. R. Momeni, "Control of a fractional-order economical system via sliding mode," Physica A, vol. 389, no. 12, pp. 2434-2442, 2010.

[45] K. Diethelm, N. J. Ford, and A. D. Freed, "A predictor-corrector approach for the numerical solution of fractional differential equations," Nonlinear Dynamics, vol. 29, no. 1-4, pp. 3-22, 2002.

[46] http://www.mathworks.com/matlabcentral/fileexchange/ 27336-fractional-order-chaotic-systems.

[47] X.-Y. Wang and J.-M. Song, "Synchronization of the fractional order hyperchaos Lorenz systems with activation feedback control," Communications in Nonlinear Science and Numerical Simulation, vol. 14, no. 8, pp. 3351-3357, 2009.

[48] A. S. Hegazi and A. E. Matouk, "Dynamical behaviors and synchronization in the fractional order hyperchaotic Chen system," Applied Mathematics Letters, vol. 24, no. 11, pp. 19381944, 2011. 


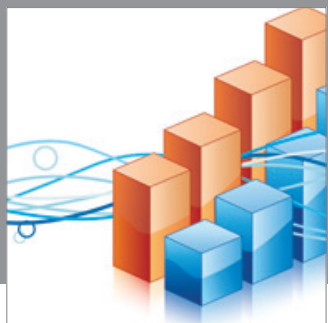

Advances in

Operations Research

mansans

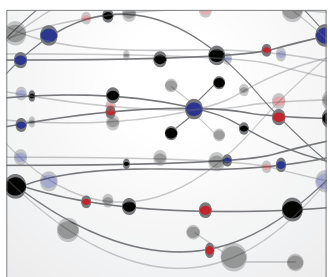

The Scientific World Journal
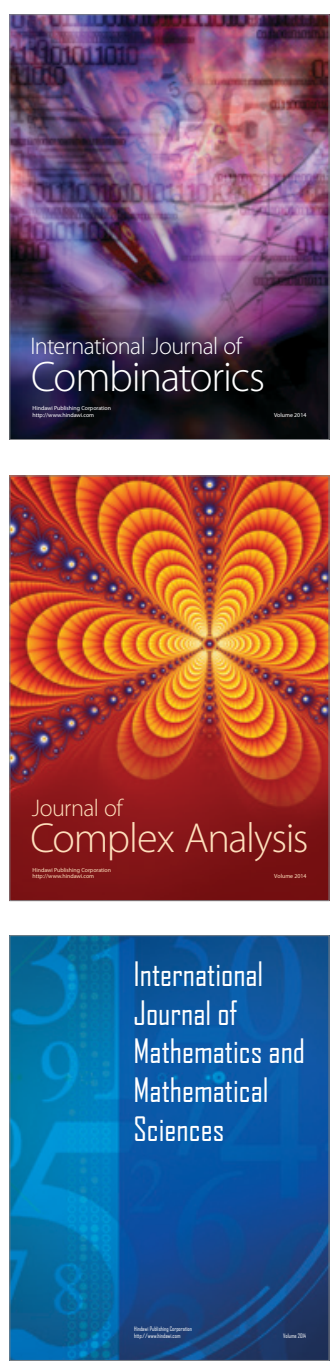
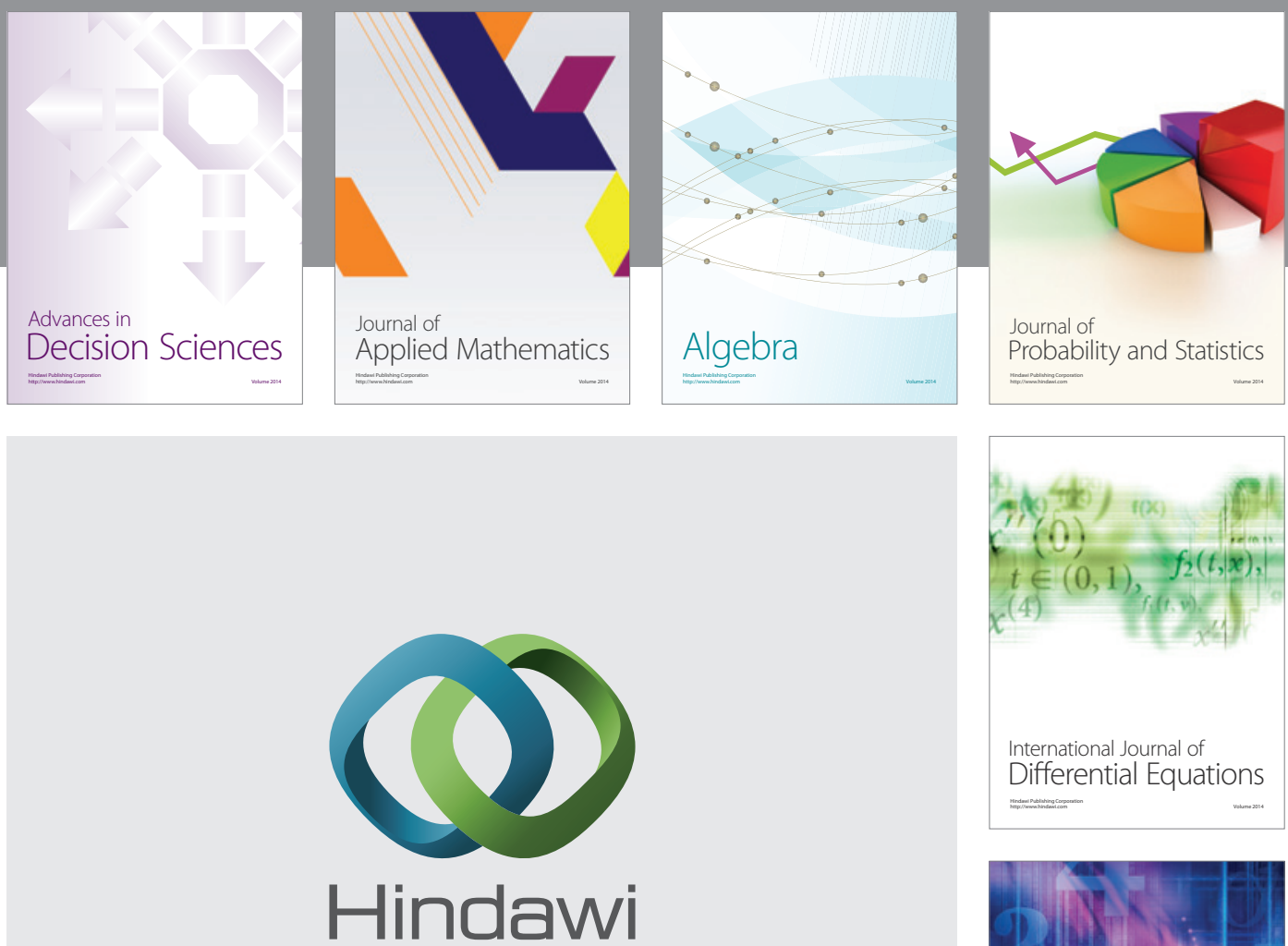

Submit your manuscripts at http://www.hindawi.com
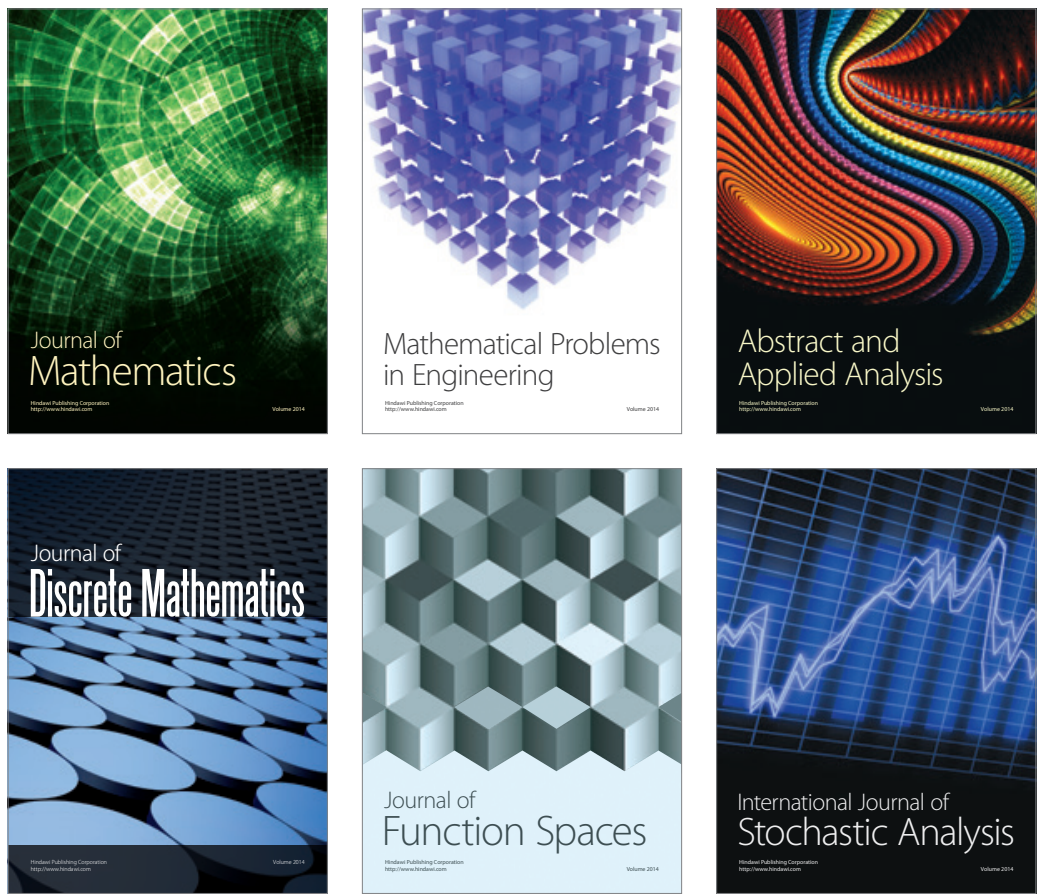

Journal of

Function Spaces

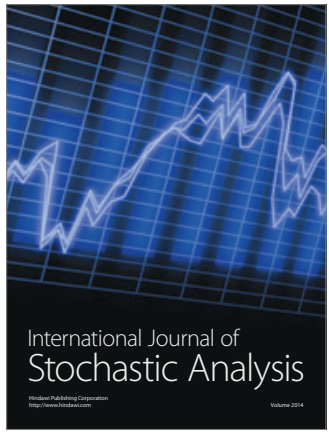

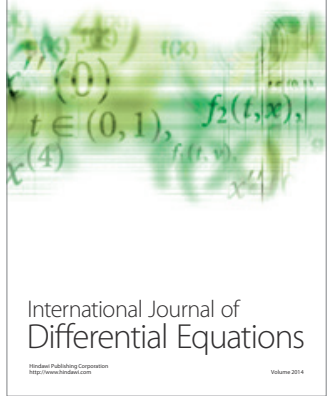
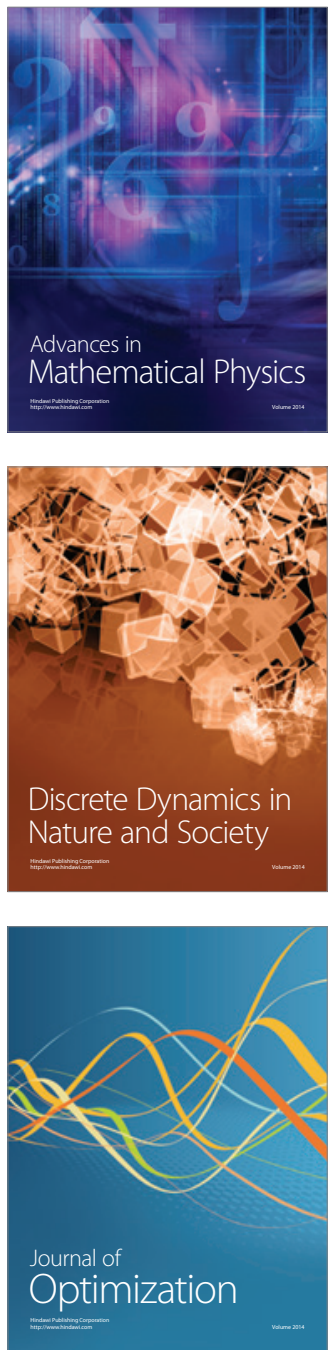